\title{
Fracture network Based on Principal Component Analysis and Neural Network- A case study in the Malay Basin
}

\author{
Shamsuddin A.A.S. ${ }^{1}$, D. Ghosh ${ }^{2}$ \\ ${ }^{1}$ Centre Subsurface of Imaging (CSI), Petronas University of Technology (Universiti Teknologi Petronas - UTP), 31750, Tronoh, Perak, \\ Malaysia \\ *Corresponding author E-mail: anassash@gmail.com
}

\begin{abstract}
Finding oil in the fractured basement rock in South East Asia has been a goal for several decades, but remains a challenge in terms of exploration/production areas in the Malay Basin due to geological complexity including fracture. Thus, the purpose of this study is to delineate fracture network based on the geometrical attributes in order to have better fracture understanding. In this study, the top of the basement acts as the key surface incorporated with the combination of geometrical seismic attributes analysis. The analysis started with data conditioning and seismic interpretation of the key surface. The final steps were conducted by using geometrical seismic attributes, principal component analysis and neural network. Principal component analysis of these four seismic attributes is able to delineate the contribution of each attributes based on eigenvalue with the PC0: 1.3450 (33.63\%), PC1:1.0556 (26.39\%), PC2:0.9270 (23.17\%) and PC3:0.6724 (16.81\%). While neural network contributes four main results (i) PC0 (ii) PC0 and PC1 (iii) PC0, PC1 and PC2 (iv) PC0, PC1, PC 2 and PC3. Fracture networks were able to be delineated and geological features that might be overlooked were able to be captured and can be used to guide the fracture network inside the fractured basement.
\end{abstract}

Keywords: fracture network; geometrical attributes; neural network; principal component analysis; self organizing map

\section{Introduction}

Fractured basement reservoirs were discovered a few decades ago in Europe, the USA, Middle East and South East Asia, including Vietnam, Indonesia and Peninsular Malaysia. In Peninsular Malaysia, fractured basement reservoirs with potential petroleum systems have been discovered in 2005 (Shahar, 2008) [1]. Fractured basements are of interest due to their reservoir complexity and heterogeneity. Geological complexities of the fractured basement reservoir were defined into rock types and tectonic regime. Basement rock was defined as any combination of metamorphic or igneous rocks without considering age, which are unconformably overlain by a sedimentary section (Landes, 1960) [2]. An additional meaning of "basement" in sedimentary origin considers the porosity value, specifically whether the rock has little and no matrix porosity (North, 1990) [3].

In this paper, a seismic attributes study was conducted in the Field A - Malay Basin. A seismic attributes is any measure of seismic data that enhanced the interpretation appearance (Satinder et al. 2007) [4]. Seismic attributes are still growing in geophysics and were developed with the objective of providing geological images for better understanding in exploration and production area. In this study, geometrical seismic attributes - variance, maximum curvature, minimum curvature and ant tracking were used as the main

geometrical analysis in finding the fracture network based on principal component analysis (PCA) and neural network analysis. Principal component analysis (PCA) is used to simplify a dataset by reducing multidimensional datasets to lower dimensions for analysis with a linear transformation that transforms the data into a new coordinate system. In the new coordinate system, the greatest variance of the data lies on the first coordinate axis (first principal component), the second greatest on the second coordinate axis, and so on.

\section{Geological Settings}

A study was conducted in the Field A, southern part of the Malay Basin. The first discovery of a fractured basement is located in the Malay Basin - an offshore part of the Peninsular Malaysia. The Malay Basin is known as an exploration matured basin and extensional origin, pull-apart basin, contains over $12 \mathrm{~km}$ of Tertiary sediment underlie with a Pre-Tertiary fractured basement (Madon et al., 1999) [5], with an estimation of basin dimension of $500 \mathrm{~km}$ length and $200 \mathrm{~km}$ width. Fractured basement reservoir known rock type ranging from metasedimentary, granite/intrusive igneous to volcanic rocks with radiometric ages of basement rocks - Jurassic to Cretaceous (Madon et al., 1999; Shahar, 2005) [5] [6].

\section{Principal Component Analysis and Neural Network Analysis}

Fracture analysis on the top of pre-Tertiary unconformity was conducted on Petrel E\&P Software Platform 2013 using seismic 3D (2004) volume, with a total area of $206 \mathrm{~km}^{2}$ was used in this study. The recording bin size of the acquisition was $6.25 \mathrm{~m} \mathrm{x}$ $18.75 \mathrm{~m}$ (crossline/inline interval) for the area. Data was acquired with a dual source, eight streamer configuration with field coverage - 64fold by using $4800 \mathrm{~m}$ cable length (Unpublished report PETRONAS, 2004) [7]. Seismic data was determined to have high 
quality in the sedimentary section up to $-2081 \mathrm{~ms}$ (two way time, twt), encountering low quality (low signal to noise ratio) as it reached inside the fractured basement. Data conditioning was performed before geometrical attributes: variance, maximum curvature, minimum curvature and ant tracking. The analysis followed by principal component analysis and neural network: supervised.

\subsection{Data Conditioning}

Seismic data conditioning is a crucial step in providing optimum seismic data quality. Data conditioning was performed before conducting seismic analysis, which includes seismic interpretation: horizon interpretation and seismic attributes analysis in order to enhance the signal in the 3D seismic migrated data. In this study, two parts of data conditioning, which are graphic equalizer and median filtering analysis were conducted. Graphic equalizer uses a frequency band filtering of the input data and median filtering replaces each sample in a window of a seismic trace by the median of the samples that fall within the analysis window. In graphic equalizer, negative values will filter frequency values while positive settings will enhance the frequencies. The purpose of a graphic equalizer is to stabilise the seismic data.

\subsection{Geometric Attributes on the Top of Basement}

The analysis was followed by using geometric attributes on the pre-Tertiary surface. In this study, four main seismic attributes variance, minimum curvature, maximum curvature and also anttracking were selected. Variance was conducted using reflectivity data based on filter length (inline and crossline length) of 5. The range of 15 vertical smoothing was conducted in this analysis. In curvature (minimum curvature and maximum curvature) constan parameter was selected based on 7 samples vertical radius, the window size is $15(7$ samples vertical radius $x 2+1)$ with 2 Inline/crossline radius: reference traces and a value of 1 chooses comparison between neighbour traces based on reflectivity data.
Training options is the maximum number of iterations, the algorithm will stop at this number even if an adequate result has not been reached. Error limit (\%) is the stop trained value when the number of points was classified incorrectly is below this limit and cross validation (\%) was applied only on supervised analysis with the percentage of the input data which is used to test the result and give the error.

\begin{tabular}{|c|c|c|c|}
\multicolumn{4}{c}{ Table 1: Neural network parameter } \\
\hline $\begin{array}{c}\text { Neural } \\
\text { network } \\
\text { class }\end{array}$ & $\begin{array}{c}\text { Max number of } \\
\text { iteration }\end{array}$ & Error Limit (\%) & Cross validation \\
\hline Supervised & 20 & 10 & 50 \\
\hline
\end{tabular}

\section{Fractured Basement - Principal Compo- nent Analysis (PCA) and Neural Network (Supervised)}

These four geometrical attributes were used as input for Principal component analysis and the results PC $0, \mathrm{PC} 1, \mathrm{PC} 2$, and PC 3 as in Fig. 1 - Fig. 4. The value of each eigenvalue can be determined as in Table 2 with PC 0 - Eigenvalue of 1.345 contributes about $33.63 \%$, PC 1 - Eigenvalue of 1.056 contributes $26.39 \%$, PC $2-$ Eigenvalue of 0.927 contributes $23.17 \%$ and PC 3 - Eigenvalue of 0.672 contributes $16.81 \%$. The value of the correlation - linear and nonlinear in between four seismic attributes can be determined as in Table 3. PCA results were used as input for neural network (supervised) with each of the contribution PCA was determined as PC 0, PC 0 and PC 1, PC 0 PC 1 and PC 2 and also PC 0 PC 1 PC 2 and PC 3 Table 3 (Fig. 5 - Fig. 8). The results were determined in two way time (twt) with display as 1 for high value and 0 for low value.

Table 2: Eigenvalue and contribution of each of Principal Component (PC)

\begin{tabular}{|c|c|c|c|c|}
\hline Correlation Coefficients & PCO & PC1 & PC2 & PC3 \\
\hline Copy of Extract value: Seismic_Migrated_Eq_Median_Variance_5x5x15 [Realized] 1 & 0.8037 & 0.0205 & 0.1268 & 0.5810 \\
\hline Copy of Extract value: Seismic_Migrated_Eq [Median filter] _3DCurv_7x2xMinCurv [Realize & -0.4647 & -0.7221 & -0.3321 & 0.3902 \\
\hline Copy of Extract value: Seismic_Migrated_Eq_Median_3DCurv_7x2xMaxCurv [Realized] 1 & 0.4098 & -0.7238 & 0.4411 & -0.3370 \\
\hline Copy of Extract value: Seismic_Migrated_Eq_Median_RAI_AntTrackingA[ [Realized] 1 & 0.5614 & -0.0987 & -0.7785 & -0.2628 \\
\hline Eigenvalue & 1.3450 & 1.0556 & 0.9270 & 0.6724 \\
\hline Contribution (\%) & 33.63 & 26.39 & 23.17 & 16.81 \\
\hline Cumulative Contribution (\%) & 33.63 & 60.01 & 83.19 & 100.00 \\
\hline
\end{tabular}

Ant Tracking was conducted based on apparent acoustic impedance data. A parameter that was selected in this work (1) Initial ant boundary: number of voxels, 7 (2) Ant track deviation, 2 (3) Ant step size: number of voxels, 3 (4) Illegal steps allowed, 1 (5) Legal criteria, 3 (6) Stop criteria 5\% and (7) Aggressive ants.

\subsection{Principal Component Analysis (PCA) and Neural Network Analysis}

PCA analysis was conducted based on four seismic attributes i) variance ii) minimum curvature iii) maximum curvature and ant tracking with the neural network was defined as supervised with a consistent maximum number of iterations was 20 , error limit was $10 \%$ and cross validation for supervised analysis was 50 (Table 1). Supervised analysis requires input-output pairs as training data. It separates the input data into distinct classes (classification), or computes an output value based on the input (estimation) by specifying the target (desired output). Three main parameters were used, including training options, error limit and cross validation. 
Table 3: Correlation - linear and nonlinear value in between four seismic attributes

Comelation type: Linear

\begin{tabular}{|c|c|c|c|c|c|}
\hline & $\begin{array}{l}\text { Copy of Extract value: } \\
\text { Seismic_Migrated_Eq } \\
\text { Median_Variance_5x5 } \\
\text { x15[Realized] } 1\end{array}$ & $\begin{array}{c}\text { Copy of Extract value: } \\
\text { Seismic_Migrated_Eq } \\
\text { [Median filter] } \\
\text { _3DCurv_7x2xMinCurv } \\
\text { [Realized] } 1\end{array}$ & $\begin{array}{l}\text { Copy of Extract value: } \\
\text { Seismic_Migrated_Eq_Medi } \\
\text { an_3DCurv_7x2xMaxCCurv } \\
\text { [Realized] } 1\end{array}$ & $\begin{array}{l}\text { Copy of Extract value: } \\
\text { Seismic_Migrated_Eq_at } \\
\text { Median_RAI_AntTrack } \\
\text { ingA[ [Realized] } 1\end{array}$ & TWT \\
\hline $\begin{array}{l}\text { Copy of Extract value: } \\
\text { Seismic_Migrated_Eq_E } \\
\text { Median_Variance_5x5x } \\
15 \text { [Realized] } 1\end{array}$ & 1.0000 & 0.2037 & 0.1747 & 0.1978 & 0.0221 \\
\hline $\begin{array}{l}\text { Copy of Extract value: } \\
\text { Seismic_Migrated_Eq } \\
\text { [Median filter] } \\
\text { 3DCurv_7×2xMinCurv } \\
\text { IRealized] } 1\end{array}$ & 0.2037 & 1.0000 & 0.0543 & 0.0336 & 0.0312 \\
\hline $\begin{array}{l}\text { Copy of Extract value: } \\
\text { Seismic_Migrated_Eq_. } \\
\text { Median_3DCurv_7x2xM } \\
\text { axCurv[Realized] } 1\end{array}$ & 0.1747 & 0.0543 & 1.0000 & 0.0467 & 0.0482 \\
\hline $\begin{array}{l}\text { Copy of Extract value: } \\
\text { Seismic_Migrated_Eq__. } \\
\text { Median_RAI_AntTracki } \\
\text { ngA[ [Realized] } 1\end{array}$ & 0.1978 & 0.0336 & 0.0467 & 1.0000 & 0.0060 \\
\hline Total & 0.3306 & 0.2233 & 0.1977 & 0.1982 & 0.0597 \\
\hline
\end{tabular}

Comelation type: Non-linear

Two highest tween minin vature and $\mathrm{v}$ were calculc well as max linear correl....

\begin{tabular}{|c|c|c|c|c|c|}
\hline & $\begin{array}{l}\text { Copy of Extract value: } \\
\text { Seismic_Migrated_Eq } \\
\text { Median__Variance_5x } \\
\text { 5x15 [Realized] } 1\end{array}$ & $\begin{array}{c}\text { Copy of Extract value: } \\
\text { Seismic_Migrated_Eq } \\
\text { [Median filter] } \\
\text { _BDCurv_7x2xMinCurv } \\
\text { [Realized] } 1\end{array}$ & $\begin{array}{l}\text { Copy of Extract value: } \\
\text { Seismic_Migrated_Eq_M } \\
\text { edian_3DCurv_7x2xMax } \\
\text { Curv[ [Realized] } 1\end{array}$ & $\begin{array}{c}\text { Copy of Extract value: } \\
\text { Seismic_Migrated_Eq_M } \\
\text { edian_RAI_AntTrackingA } \\
\text { [[ [Realized] } 1\end{array}$ & TWT \\
\hline $\begin{array}{l}\text { Copy of Extract value: } \\
\text { Seismic_Migrated_Eq_M } \\
\text { edian_Variance_5x5x15 } \\
\text { [Realized] } 1\end{array}$ & 1.0000 & 0.3040 & 0.1119 & 0.1157 & 0.0016 \\
\hline $\begin{array}{l}\text { Copy of Extract value: } \\
\text { Seismic_Migrated_Eq } \\
\text { [Median filter] } \\
\text { 3DCurv_7x2xMinCurv } \\
\text { [Realized] } 1\end{array}$ & 0.3040 & 1.0000 & 0.2395 & 0.0052 & 0.0136 \\
\hline $\begin{array}{l}\text { Copy of Extract value: } \\
\text { Seismic_Migrated_Eq_M } \\
\text { edian_3DCurv_7x2xMax } \\
\text { Curv[Realized] } 1\end{array}$ & 0.1119 & 0.2395 & 1.0000 & 0.0322 & 0.0791 \\
\hline $\begin{array}{l}\text { Copy of Extract value: } \\
\text { Seismic_Migrated_Eq_M } \\
\text { edian_RAI_AntTrackingA } \\
\text { [ [Realized] } 1\end{array}$ & 0.1157 & 0.0052 & 0.0202 & 1.0000 & 0.0062 \\
\hline Total & 0.3744 & 0.4108 & 0.3083 & 0.1204 & 0.0807 \\
\hline
\end{tabular}
curvature and maximum curvature.

Two main geological patterns were observed in principal component analysis. The high value of principal component analysis 1 able to delineate geological features. First geological patterns (zone 1) were observed in principal component 1, 2, 3 and 4 as in orange circle (Fig.1 - Fig.4). Second geological patterns (zone 2) were observed in in principal component 1,2,3 and 4 as in yellow circle (Fig.1 - Fig.4). First geological patterns (zone 1) were determined as a system while second geological patterns were determined oriented in east-west.

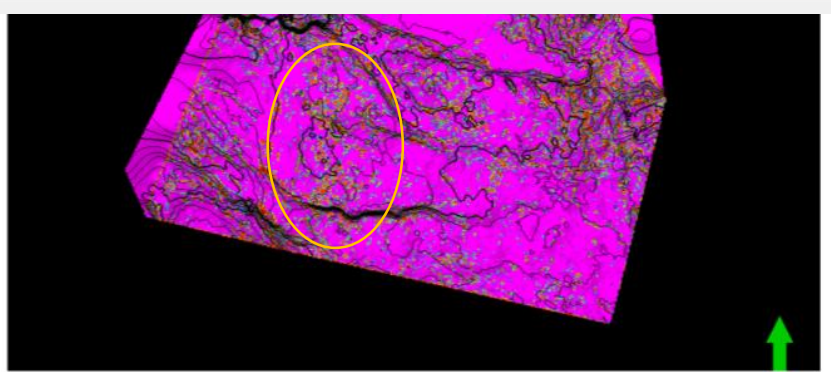

Fig.1: Principal Component 1 (PC 1)

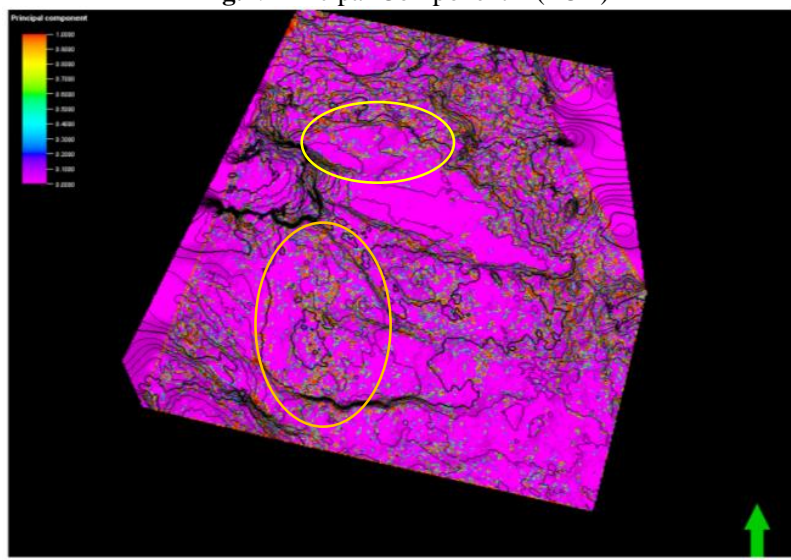

Fig. 2: Principal Component 2 (PC 2) 


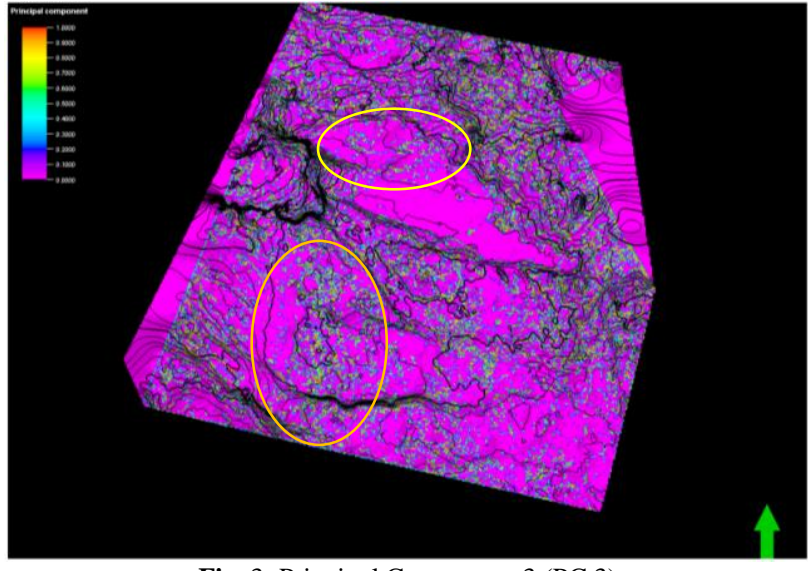

Fig. 3: Principal Component 3 (PC 3)

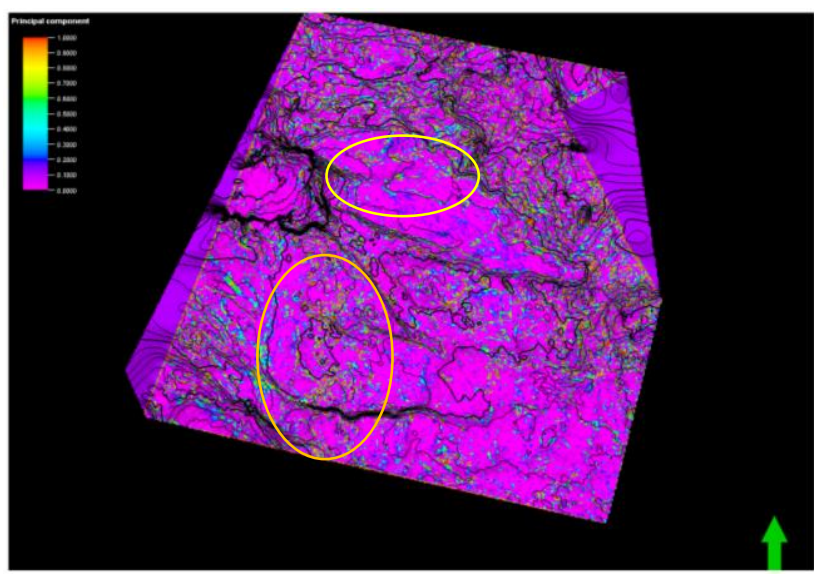

Fig. 4: Principal Component 4 (PC 4)

Four results were able to be produced by using neural network based on supervised analysis. They represent different geological complexities of the fractured basement reservoir. The geological complexities were observed to be increased as the input of principal component analysis increase from PC 0 to PC 0, PC 1 , PC 2, PC 3 and PC 4. Geological features in zone 1 (purple circle) were observed in different ranges of two way time as in Fig.6, Fig. 7 and Fig. 8. In zone 2 (red circle), neural network based on supervised analysis is able to delineate separated geological features in different ranges of two way time as in the Fig.6, Fig. 7 and Fig. 8. Neural network based on supervised by using PC 0 was observed to have less information in zone 1 and zone 2 compared to the other analysis.

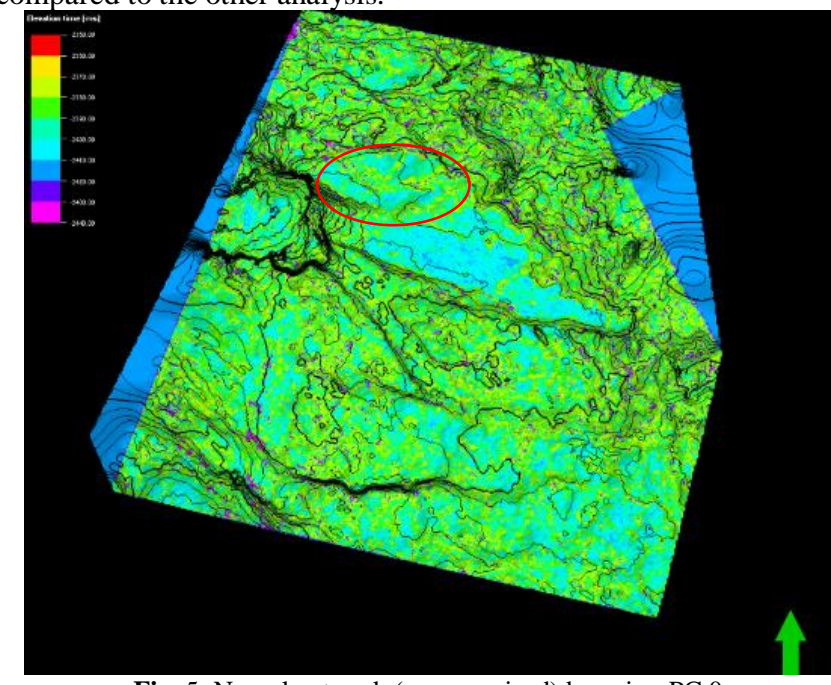

Fig. 5: Neural network (unsupervised) by using PC 0

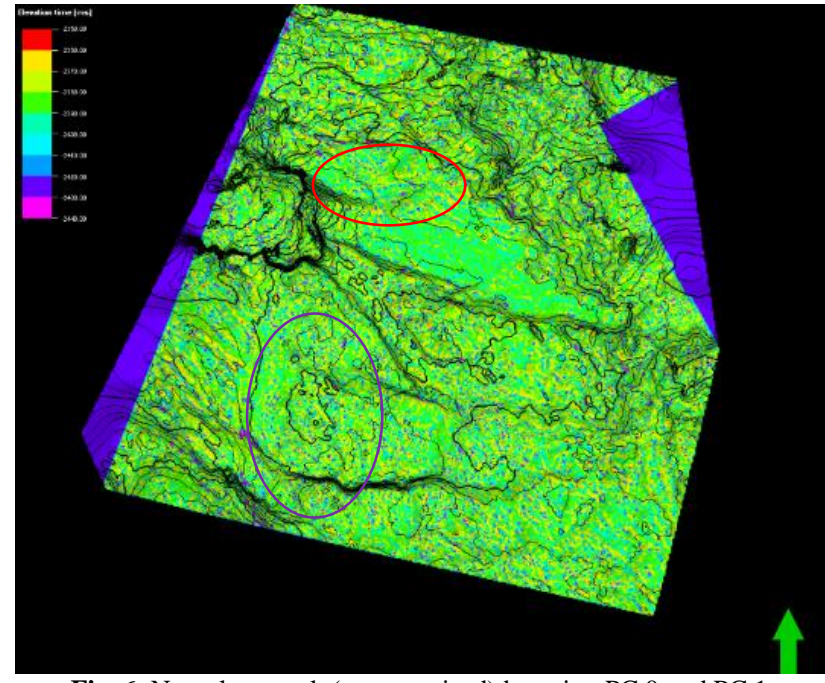

Fig. 6: Neural network (unsupervised) by using PC 0 and PC 1

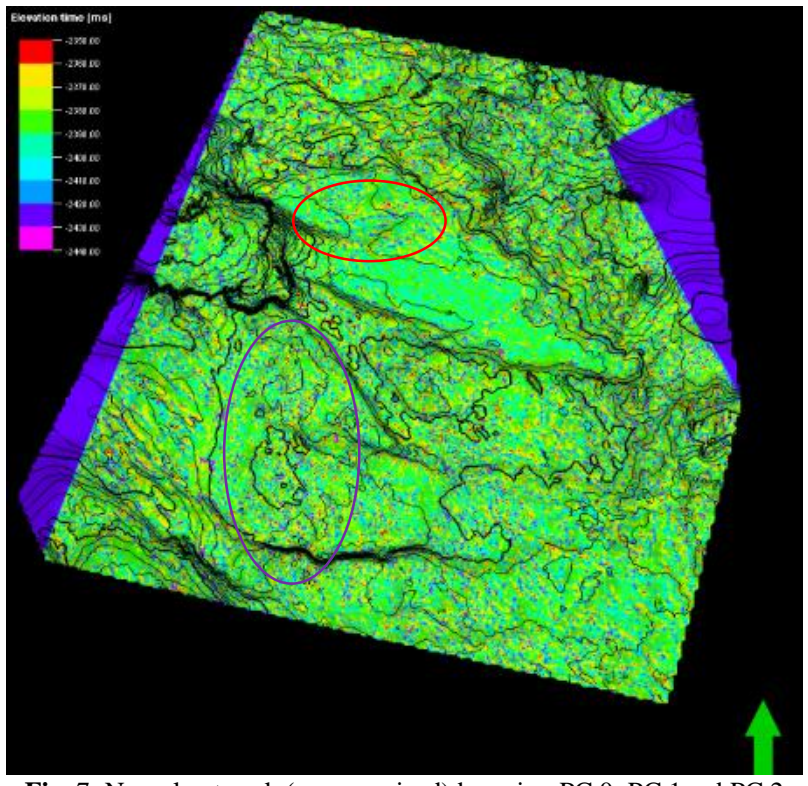

Fig. 7: Neural network (unsupervised) by using PC 0, PC 1and PC 2

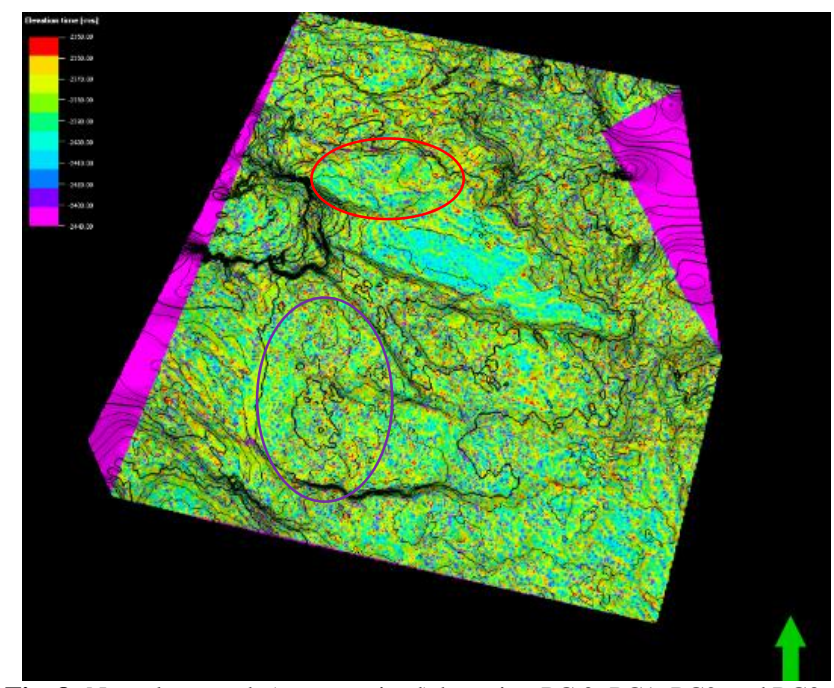

Fig. 8: Neural network (unsupervised) by using PC 0, PC1, PC2 and PC3

\section{Discussion}

The similarity of the geological patterns can be seen at the top of pre-Tertiary in two main zones. High value in the principal component analysis were able to delineate geological features. The 
geological features that were delineated are suggested as fracture network. The delineation of fracture network is supported by using neural network based on unsupervised analysis. Principal component analysis, zone 1 was observed as a fracture network in the system and zone 2 was observed as fracture network with the orientation of east-west. Neural network analysis based on supervised is able to determine four different of fractured basement reservoir complexities. Principal component analysis is able to delineate fracture network incorporated by detailed fractured network that were observed in neural network based on supervised analysis. Fracture networks were able to be delineated and geological features that might be overlooked were able to be captured by using principal component analysis and neural network (supervised). It can be used to guide the fracture network inside the fractured basement

\section{Conclusion}

Geometrical attributes were able to be simplified by using principal component analysis and neural network supervised. Fracture networks on the top of pre-Tertiary were able to be delineated. Correct input in PCA / Neural Network was emphasised in this study align with the suggestion by Tao Zhao et al., 2015 [8] in order to have geological meaning, especially in detecting structural. The appearance of geological features that might be overlooked was able to be captured by the PCA and Neural Network with supervised analysis. These analysis were able to capture the possibilities of fracture network in detail on the top of the basement.

\section{Acknowledgement}

The authors would like to thank the sponsors of the project, PETRONAS for research funding and permission to publish the work. We also wish to thank Centre of Excellence in Subsurface Seismic Imaging \& Hydrocarbon Prediction (CSI) and Universiti Teknologi PETRONAS for research facilities.

\section{References}

[1] Shahar, S. 2008, Structural evolution of the Tenggol Arch and its implication for basement fracture patterns in the Malay Basin, Malaysia, Durham theses, Durham University.

[2] Landes KK., Charlesworth J.R., Heany F., Lesperance P.J.. 1960 , Petroleum Resources in Basement Rocks, Bull. AAPG, 44, 1682 1691.

[3] North FK. 1990, Petroleum Geology. Second Ed.:Winchester, Mass, Unwin Hyman Ltd, $631 \mathrm{pp}$

[4] Satinder C., Marfurt KJ.. 2007, Seismic Attributes for prospect identification and reservoir characterization. Tulsa, OK, U.S.A

[5] Madon, M.B., Abolins, P., Hoesni, M.J.B., Ahmad, M.B., 1999 Malay basin. In: Selley, R., Meng, L.K. (Eds.), The Petroleum Geology and Resources of Malaysia. Petronas, Kualu Lumpur, pp. 173 $-217$

[6] Shahar, S 2005 The prospectivity of Fractured Basement Play of the Malay Basin, Petroleum Geology Conference and Exhibition, pp 42

[7] Unpublished report PETRONAS, 2004. Report of Seismic Data Processing

[8] Tao Z., Vikram J., Atish R., Marfurt KJ. 2015 "A comparison of classification techniques for seismic facies recognition." Interpretation, 3(4), SAE29-SAE58. 